\title{
Theory of High-Tc Superconducting Cuprates Based on Experimental Evidence
}

\author{
A.A. Abrikosov \\ Materials Science Division, Argonne National Laboratory \\ 9700 South Cass Avenue, Argonne, Illinois 60439, USA
}

(September 19, 2018)

\begin{abstract}
A model of superconductivity in layered high-temperature superconducting cuprates is proposed, based on the extended saddle point singularities in the electron spectrum, weak screening of the Coulomb interaction and phonon-mediated interaction between electrons plus a small short -range repulsion of Hund's, or spinfluctuation, origin. This permits to explain the large values of $T c$, features of the isotope effect on oxygen and copper, the existence of two types of the order parameter, the peak in the inelastic neutron scattering, the positive curvature of the upper critical field, as function of temperature etc.
\end{abstract}

PACS numbers:74.20.Fg,74.25.Kc,74.62.Dh,74.72.Bk

\section{PHONON-MEDIATED INTERACTION}

The origin and the symmetry of the order parameter in the HTSC is the primary topic in the physics of high-temperature layered cuprates. The crystalline symmetry, even if it is assumed tetragonal, and the order parameter to be real (this follows from time-reversal symmetry), requires only that at a $\pi / 2$ rotation in the ab-plane it either does not change, or changes its sign. Symmetry by itself does not tell us how many nodes must exist in either case, or whether the formula $\cos k_{x}-\cos k_{y}$ is correct. Therefore the only way to find the order parameter is to study the interaction between electrons, which leads to pairing. The high values of Tc and absence of the isotope effect in optimally doped $\mathrm{YBa}_{2} \mathrm{Cu}_{3} \mathrm{O}_{7-\delta}$ (YBCO) led to the conclusion that this interaction cannot be mediated by phonons. However the experiments by the groups of J. Franck and D. Morris [1, 2] showed that a partial substitution of $Y$ by $P r$, or of $B a$ by $L a$ leads to the increase of the isotope effect simultaneously with the decrease of the critical temperature, so that at $T c \approx 40 K \alpha \approx 0.4\left(T_{c} \propto M^{-\alpha}\right)$ (Fig.1). An alternative appears: either these substituted compounds have a different mechanism of superconductivity compared to YBCO with $T c \approx 90 K$, or the mechanism is always phonons, and the absence of the isotope effect in YBCO is due to something else. I prefer the last option. In its favor speak also the tunneling spectra at higher voltages in $\mathrm{Nd}_{2-x} \mathrm{Ce}_{x} \mathrm{CuO} \mathrm{O}_{4}$ (NCCO) [3] (Fig.2) and in $\mathrm{Bi}_{2} \mathrm{Sr}_{2} \mathrm{CaCu}_{2} \mathrm{O}_{8}$ (BSCCO) [4] (Fig.3). The Eliashberg interaction constant $\alpha^{2} F(\omega)$ extracted from the tunneling conductance has a shape corresponding to the phonon density of states $F(\omega)$ obtained from inelastic neutron scattering. The deep minima in $\alpha^{2} F(\omega)$ compared to $F(\omega)$ can be attributed to the fact that electrons interact differently with different phonons. Lately the strong electron-phonon interaction in BSCCO was confirmed by appearance of branching on the current-voltage characteristic of a break junction at $\mathrm{eV}<2 \Delta$ [5], representing phonon-assisted Josephson effect (similar to Shapiro steps) for low-frequency optical phonons. The frequencies fitted the Raman data (see sec. 6).

\section{EXTENDED SADDLE POINT SINGULARITIES}

A few years ago two experimental groups: J.-C. Campuzano's from Argonne [G] and Z.-X.Shen's from Stanford [7], investigating angle resolved photoemission spectra (ARPES), discovered that the dependence of the energy of quasiparticles on their momentum $\epsilon\left(k_{x}, k_{y}\right)$ (the dependence on $k_{z}$ is weak due to quasi-two-dimensionality) has flat regions, where it does not depend on one of the components, so that the spectrum becomes quasi-one-dimensional in these regions (Fig.4). This was first discovered in YBCO, $\mathrm{YBa}_{2} \mathrm{Cu}_{4} \mathrm{O}_{8}$, BSCCO, and appeared also in band structure calculations by several groups, particularly, A.Freeman's [8], for mercury compounds. Such a feature in the spectrum was called "extended saddle point singularity". The density of states in the vicinity of such a singularity is proportional to $\left(\epsilon-\epsilon_{0}\right)^{-1 / 2}$. If the Fermi energy is close to $\epsilon_{0}$, this enhancement is more than sufficient to explain the high values of Tc assuming the usual strength of the electron-phonon interaction.

The second feature is that the integral entering the BCS equation

$$
\Delta(\mathbf{p})=\int K\left(\mathbf{p}, \mathbf{p}^{\prime}\right) f\left[\Delta\left(\mathbf{p}^{\prime}\right)\right] \frac{d^{3} \mathbf{p}^{\prime}}{(2 \pi)^{3}}
$$

which is logarithmically divergent, if the density of states is constant, becomes convergent. In the case, if $\epsilon_{F}-\epsilon_{0} \ll$ $\omega_{0}, \omega_{0}$ being some characteristic phonon frequency, the critical temperature does not depend on $\omega_{0}$, and hence there is no isotope effect. The BCS-type formula for $T c$ for this case has the form:

$$
T_{c}=\frac{8 \gamma\left(\epsilon_{F}-\epsilon_{0}\right)}{\pi} \exp \left[-\frac{4 \sqrt{2} \pi^{2}(n-1)\left(\epsilon_{F}-\epsilon_{0}\right)}{g m_{x}^{1 / 2} \kappa^{2}}\right]
$$

The most important here is the appearance of $\epsilon_{F}-\epsilon_{0}$ in the exponent and in the prefactor (I will not explain the meaning of other notations in this expression). If the distance $\epsilon_{F}-$ $\epsilon_{0}$ increases, $T_{c}$ decreases, and, at the same time, $\epsilon_{F}-\epsilon_{0}$ in the prefactor is gradually replaced by $\omega_{0}$, enhancing the isotope effect [9]. This fits the experimental data. I would like 
to stress that the usual saddle point singularity, extensively studied by several groups, e.g. J. Friedel, A. Labbe, J. Bok, D. Newns, R. Markievicz (see [10] for references) does not lead to such consequences. The enhancement of Tc is weak, and no new energy scale is introduced in the BCS integral, so that the absence of the isotope effect cannot be explained. Therefore the statement that the flat regions are equivalent to $2 \mathrm{D}$ van Hove singularities is wrong.

\section{BAD COULOMB SCREENING}

Under proper assumptions the flat region can dominate in the total density of states; then, however, we encounter another puzzle. The ARPES experiments of the same groups established a marked anisotropy of the energy gap (in the BCS theory the gap corresponds to the absolute value of the order parameter). The usual belief is that with phonon-mediated interaction no anisotropy of the order parameter can be obtained, even in case if the energy spectrum of the metal is anisotropic. This is due to exchange of phonons with high momenta which effectively connect all points of the Fermi surface in the BCS equation (1) (see Fig.5). However, this statement, being true for good metals, is not so evident for the substances under consideration. According to numerous expe-rimental data, they are intermediate between metals and ionic crystals. The cross-over between these two limiting cases can be imagined as the change of the Debye screening radius from interatomic distances to infinity, and the HTSC can be viewed as substances where all Coulomb-based interactions are screened at large distances. This refers also to the electron attraction mediated by phonons, since it is due to the electron-ion interaction. Therefore it is reasonable to assume this interaction in the form

$$
V(\mathbf{k})=g\left(\frac{\kappa^{2}}{k^{2}+\kappa^{2}}\right)^{n} \frac{\omega^{2}(k)}{\left(\xi-\xi^{\prime}\right)^{2}-\omega^{2}(k)}
$$

where $\kappa \ll K$ is the reciprocal Debye radius, $\xi$ is the electronic energy, and $K$ - the reciprocal lattice period. Since different models lead to different powers $n$, we will simply assume $n>1$. Under such circumstances the exchange of phonons with small momenta becomes dominant, and we cannot reach the singular region from some distant place (Fig.5) without decreasing drastically the value of the order parameter. Therefore it becomes very anisotropic, and its maxima are located in the regions with the maximal density of states [11]; this, in fact, is observed in experiment. Moreover the values, distant from the singular regions, will be defined by the maximal value, the connection being established through the BCS equation. Therefore the critical temperature is defined only by these singular regions.

\section{TWO TYPES OF THE ORDER PARAMETER}

In a purely phonon picture there can be no changes of sign of the order parameter, and this contradicts many reliable experimental data. However, apart from phonons, there can exist interactions of some other origin. The isotope effect tells us that the phonons dominate. Therefore these other interactions can become important only, if their nature differs substantially from the phonon-mediated attraction. We will assume that this additional interaction is a short-range repulsion coming either from spin fluctuations, or being the renormalized repulsion at the $\mathrm{Cu}$ sites. To the first approximation different regions with the maximal density of states and the maximal absolute value of the order parameter are disconnected. Therefore, the order parameter can be either even, or odd with respect to a $\pi / 2$ rotation in the ab-plane. The corresponding formulas are:

$$
\begin{array}{r}
\frac{\Delta(\varphi)}{\Delta_{1}}=\frac{2(n-1) P_{0 y}}{\kappa^{2} d} \times \\
\left\{\left(\frac{\kappa}{2 p_{0}}\right)^{2 n}\left[\sin ^{-2 n}\left(\frac{\varphi}{2}\right)+\sin ^{-2 n}\left(\frac{\varphi-\varphi_{0}}{2}\right)\right]-\frac{U}{g}\right\} \\
{\left[\sin ^{-2 n}\left(\frac{\varphi}{2}\right)-\sin ^{-2 n}\left(\frac{\left|\varphi-\varphi_{0}\right|}{2}\right)\right]}
\end{array}
$$

where $\Delta_{1}$ is the order parameter in the singular region, $P_{0 y}$ is the length of the singularity, $d$ is the period in the cdirection, $p_{0}$ is the radius of the Fermi surface, which we assume cylindrical, 0 and $\varphi_{0}=\pi / 2$ are the locations of singularities, and $U$ is the amplitude of the short-range repulsion, which we suppose isotropic. These expressions are valid far from the singular regions. A simple interpolation permits to describe the whole Fermi surface. The simplest formulas for $n=1$ are

$$
\begin{aligned}
\frac{\Delta_{\text {even }}}{\Delta_{1}} & =\frac{A}{A+1-\cos (4 \varphi)}, \\
\frac{\Delta_{\text {odd }}}{\Delta_{1}} & =\frac{A \cos (2 \varphi)}{A+1-\cos (4 \varphi)}
\end{aligned}
$$

(A-adjustable constant), representing the even and odd cases. Fig. 6 presents the plot of eq. (7) for $A=1$. The order parameters (4) have the same symmetry, as the"s-wave" and "dwave" although they differ from the usual expressions. As already said, to the first approximation the even and odd states are degeneratre. This degeneracy is lifted, if we take into account the energy of the small overlap of the order parameter originating from different singular regions. In case, if there were only phonon attraction, the even solution would have definitely the lowest energy. However, the presence of a small short-range repulsion with an isotropic Fourier amplitude in momentum space can drastically change the situation [12]. 
The interaction Hamiltonian in momentum representation has the shape:

$$
\begin{array}{r}
H_{i n t}=-\frac{g}{2} \sum_{\mathbf{p}_{\mathbf{1}} \mathbf{p}_{\mathbf{2}} \mathbf{k}} a_{\alpha}^{\dagger}\left(\mathbf{p}_{\mathbf{1}}\right) a_{\beta}^{\dagger}\left(-\mathbf{p}_{\mathbf{1}}+\mathbf{k}\right) \times \\
V\left(\mathbf{p}_{\mathbf{1}}-\mathbf{p}_{\mathbf{2}}\right) a_{\beta}\left(-\mathbf{p}_{\mathbf{2}}+\mathbf{k}\right) a_{\alpha}\left(\mathbf{p}_{\mathbf{2}}\right) \\
+\frac{U}{2} \sum_{\mathbf{p}_{\mathbf{1}} \mathbf{p}_{\mathbf{2}} \mathbf{k}} a_{\alpha}^{\dagger}\left(\mathbf{p}_{\mathbf{1}}\right) a_{\beta}^{\dagger}\left(-\mathbf{p}_{\mathbf{1}}+\mathbf{k}\right) a_{\beta}\left(-\mathbf{p}_{\mathbf{2}}+\mathbf{k}\right) a_{\alpha}\left(\mathbf{p}_{\mathbf{2}}\right)
\end{array}
$$

where $V(\mathbf{k})=\left[\kappa^{2} /\left(k^{2}+\kappa^{2}\right)\right]^{n}$. Since the main contribution comes from vicinities of the singular regions, which are in the a- and b- directions, we separate parts, associated with the condensate by putting $k=0$ and transforming

$$
a_{\alpha}^{\dagger}\left(\mathbf{p}_{\mathbf{1}}\right) a_{\beta}^{\dagger}\left(-\mathbf{p}_{\mathbf{1}}\right) \rightarrow T \sum_{\omega_{n}} F^{\dagger}\left(\omega_{n}, \mathbf{p}_{\mathbf{1}}\right) I_{\alpha \beta} \equiv \tilde{F}^{\dagger}\left(-\mathbf{p}_{\mathbf{1}}\right) I_{\alpha \beta}
$$

where $I_{\alpha \beta}$ is the unit antisymmetric matrix: $I=i \sigma_{y}$. The $\tilde{F}^{\dagger}$ can be considered as a creation operator of a Cooper pair, consisting of electrons with momenta $\mathbf{p}_{\mathbf{1}}$ and $-\mathbf{p}_{\mathbf{1}}$. We leave quadratic and linear terms in $\tilde{F}^{\dagger}$. Then we get

$$
\begin{array}{r}
H_{\text {int }} \approx-\frac{2 P_{y 0}}{(2 \pi)^{2} v_{1} d}\left[\lambda_{1}\left(\Phi_{a}^{2}+\Phi_{b}^{2}\right)+2 \lambda_{2} \Phi_{a} \Phi_{b}\right] \\
-\frac{\lambda_{1}}{2}\left[\sum_{\mathbf{p} \in \mathbf{a}} \Phi_{a} I_{\alpha \beta}\left(a_{\beta}(\mathbf{p}) a_{\alpha}(-\mathbf{p})+a_{\alpha}^{\dagger}(-\mathbf{p}) a_{\beta}^{\dagger}(\mathbf{p})\right)+\right. \\
\left.\sum_{\mathbf{p} \in \mathbf{b}} \Phi_{b} I_{\alpha \beta}\left(a_{\beta}(\mathbf{p}) a_{\alpha}(-\mathbf{p})+a_{\alpha}^{\dagger}(-\mathbf{p}) a_{\beta}^{\dagger}(\mathbf{p})\right)\right] \\
-\frac{\lambda_{2}}{2}\left[\sum_{\mathbf{p} \in \mathbf{b}} \Phi_{a} I_{\alpha \beta}\left(a_{\beta}(\mathbf{p}) a_{\alpha}(-\mathbf{p})+a_{\alpha}^{\dagger}(-\mathbf{p}) a_{\beta}^{\dagger}(\mathbf{p})\right)\right. \\
\left.-\sum_{\mathbf{p} \in \mathbf{a}} \Phi_{b} I_{\alpha \beta}\left(a_{\beta}(\mathbf{p}) a_{\alpha}(-\mathbf{p})+a_{\alpha}^{\dagger}(-\mathbf{p}) a_{\beta}^{\dagger}(\mathbf{p})\right)\right]
\end{array}
$$

where

$$
\begin{array}{r}
\lambda_{1}=\frac{1}{(2 \pi)^{2} v_{1}}\left(\frac{g \kappa^{2}}{n-1}-\frac{2 U P_{y 0}}{d}\right), \\
\lambda_{1}=\frac{2 P_{y 0}}{(2 \pi)^{2} v_{1}}\left[g\left(\frac{\kappa^{2}}{2 p_{0}^{2}}\right)^{n}-U\right] \\
\Phi_{a, b}=\int_{-\mu_{1}}^{\infty}\left(\frac{\mu_{1}}{\xi+\mu_{1}}\right)^{1 / 2} \tilde{F}_{a, b}(\xi) d \xi
\end{array}
$$

We used here that in the singular region the functions $\tilde{F}_{a, b}$ depend only on the normal component of the momentum, i.e., on $\xi=v_{1}\left(p_{n}-p_{0}\right)$, and performed integrations over other components. Since we assume that $g \kappa^{2} \gg U P_{y 0} / d$ and $\kappa \ll p_{0}$, we have: $\lambda_{1} \gg \lambda_{2}$, and hence the overlap terms in the Hamiltonian are indeed small. We would like to stress here that the reduced interaction Hamiltonian (10), where only the singular momentum regions were left, is sufficient for calculation of the critical temperature and total energy but fails when the low-energy excitations play the major role, as in the specific heat and kinetics at low temperatures. Applying all the superconducting machinery (see ref. [13]) to the full Hamiltonian, including kinetic terms, we obtain the necessary quantities:

$$
\begin{array}{r}
T_{c, \text { odd }}=8 \frac{\gamma}{\pi} \mu_{1} \exp \left[-1 /\left(\lambda_{1}-\lambda_{2}\right)\right] \\
=\frac{\gamma}{\pi} \Delta_{1} \exp \left\{-\lambda_{2} /\left[\lambda_{1}\left(\lambda_{1}-\lambda_{2}\right)\right]\right\} \\
\approx \frac{\gamma}{\pi} \Delta_{1} \exp \left(-\lambda_{2} / \lambda_{1}^{2}\right) \\
T_{c, \text { even }} \simeq \frac{\gamma}{\pi} \Delta_{1} \exp \left(\lambda_{2} / \lambda_{1}^{2}\right), \lambda_{2} \ll \lambda_{1} \\
T_{c, \text { odd }}-T_{c, \text { even }} \approx-2 \frac{\gamma}{\pi} \Delta_{1} \sinh \left(\lambda_{2} / \lambda_{1}^{2}\right), \\
\Delta_{1}=8 \mu_{1} \exp \left(-1 / \lambda_{1}\right) \\
\Omega_{\text {odd }}(0)-\Omega_{\text {even }}(0) \approx \frac{4 P_{y 0}}{(2 \pi)^{2} v_{1} d} \Delta_{1}^{2} \sinh \left(\lambda_{2} / \lambda_{1}^{2}\right)
\end{array}
$$

From (12) and (13) it can be seen that, if $\lambda_{2}>0$, the equilibrium solution is even, and, if $\lambda_{2}<0$, it is odd. This occurs at all temperatures, where superconductivity exists. From the definitions (13) it follows that the phonons dominate in the interaction, if

$$
g \kappa^{2} /(n-1) \gg 2 U P_{y 0} / d
$$

Under this condition $\lambda_{2}$ can have either sign. It is negative, if

$$
U>g\left[\kappa^{2} /\left(2 p_{0}^{2}\right)\right]^{n}
$$

The main conclusion is the division of layered cuprates in two very distinct groups. Strong anisotropy, originating from extended saddle point singularities and a long screening radius, makes it plausible that the order parameter becomes odd, whereas weak anisotropy leads to an even order parameter. Since the quasi-1D energy spectrum leads to a singularity in the density of states only from one side, it is likely to be absent in the n-type cuprates, as NCCO . This can be the origin of their lower $T_{c}$ and of the even order parameter.

\section{SCATTERING FROM IMPURITIES}

A strong proof of the odd order parameter is the action of impurities [14]. Regular substitutional impurities are most likely ionized, and being small-angle scatterers, they do not influence $T_{c}$ substantially. Of course, there is also some largeangle scattering but it has a small probability. The usual estimates lead to the conclusion that the critical temperature is influenced only if $\tau \epsilon_{F} \sim 1$, which could be considered in favor of s-pairing. The fault of such an estimate is that a much larger $\tau$ should be taken, namely, that of large angle scattering, and it could fit the condition $\tau \Delta \sim 1$. In the experiment [15], defects were created in YBCO by electron bombardment. The electron energy was chosen so that the oxygen atoms were displaced but not knocked out; this produced dipolar defects causing large-angle scattering. They were very effective in 
suppression of the critical temperature. At the same time the copper atoms were not involved, and this excluded magnetic defects. The observed dependence of Tc on the concentration of these defects fits the prediction for an odd order parameter:

$T_{\mathrm{co}} \approx \begin{cases}T_{c o}^{\text {clean }}\left[1-\pi /\left(2 T_{c o}^{\text {clean }} \tau\right)\right], & \left(\pi T_{c o}^{\text {clean }} \tau\right)^{-1} \ll 1 \\ \frac{2 \sqrt{6}}{\pi} \sqrt{\tau_{c}^{-1}\left(\tau_{c}^{-1}-\tau^{-1}\right)}, & \left(\tau_{c}^{-1}-\tau^{-1}\right) \ll \tau_{c}^{-1}\end{cases}$

where $\tau^{-1}$ is the scattering probability proportional to the residual resistance in the normal state, and

$$
\tau_{c}^{-1}=(\pi / 4 \gamma) T_{c o}^{(\text {clean })}
$$

is the critical value, where $T_{c o}$ vanishes. In reality, however, this does not happen, because the metal can undergo a phase transition into the even phase. This was not observed in the experiment [15] in the accessible temperature interval, however there exist other ways to suppress the d-wave order parameter, and at least in one of such experiments performed by the group of L. Greene a "subdominant" s-wave order parameter was indeed observed [16].

\section{NEGATIVE ISOTOPE EFFECT ON COPPER}

Rather close considerations can explain the negative isotope effect on copper, discovered by the Franck's group [17]. It was found that in underdoped samples of YBCO the substitution ${ }^{63} \mathrm{Cu}-{ }^{65} \mathrm{Cu}$ causes a change of $T_{c}$ corresponding to a negative isotope effect, namely, a larger copper isotope mass leads to an increase of the critical temperature. There exist some other examples of a negative isotope effect, e. g., resulting from a substitution of hydrogen by deuterium in solid solutions. The distinction of the present case is that the same substances have a positive isotope effect for the oxygen substitution ${ }^{16} \mathrm{O}-{ }^{18} \mathrm{O}$. The absolute value of the negative copper isotope effect depends on the oxygen concentration. In optimally doped samples it is small, otherwise it depends nonmonotonically on concentration. The maximal absolute value is around 0.4 ; we have in mind the power a in the dependence $T c \propto M^{-\alpha}$, or for small variations of $\mathrm{M}$,

$$
\alpha=-\Delta\left(\ln T_{c}\right) / \Delta(\ln M) .
$$

In a simple model one cannot hope to reproduce all the details, and our goal will be only the qualitative explanation of the most spectacular features. All phonons, whatever their nature is, contribute to the attractive interaction, and therefore, in order to explain the negative isotope effect, we have to think about some different action. In this connection we may remember that in a d-wave superconductor ordinary potential nonmagnetic scatterers with large angle scattering suppress superconductivity. Let us suppose that there exist phonons which do not affect strongly the overall ionic charge. The interaction of electrons with these phonons will be short-ranged, the same as with neutral defects. If we suppose also that the characteristic frequency of such phonons is small compared to $T c$, we obtain a situation similar to elastic impurity scattering. In a d-wave superconductor this scattering will suppress superconductivity, and its effect will increase with increasing amplitude of oscillations, i. e. with decreasing mass of the ions involved. This consideration is indeed justified by exact calculations. The result is

$$
\alpha \approx-6.326 g_{2}^{2} \nu\left(\frac{\omega_{02}}{2 \pi T_{c}}\right)^{2}
$$

where $\omega_{02}$ and $g_{2}^{2}$ are the frequency of $\mathrm{Cu}$ phonons with momentum $p_{0} \sqrt{2}$ and their interaction constant with electrons, $\nu$ is the density of states. The effect increases in magnitude with decreasing $T_{c}$, i. e. with underdoping. For an s-type order parameter the isotope effect is always positive. The Raman scattering experiments [18], [19] show that indeed low frequency phonons do exist. The best candidate is the mode with $\omega_{0} \approx 150 \mathrm{~cm}^{-1}$ which corresponds to vibrations of $\mathrm{Cu}$ atoms. For this mode the expansion parameter of the theory $\left(\omega_{0} / 2 \pi T_{c}\right)^{2} \sim 0.24$ (we took $\left.T_{c}=70 K\right)$.

\section{NEUTRON PEAK}

Another interesting consequence of the proposed theory is the explanation of the origin and the prediction of the fine structure of the peak in inelastic neutron scattering in YBCO [20]. The clear narrow peak was observed in the superconducting phase for momentum change close to $(\pi / 2, \pi / 2)$ and energy change close to $41 \mathrm{meV}$ ( [21], and references thereon), which corresponds, approximately, to $2 \Delta_{\max }$, as obtained from tunneling measurements [22]. The momentum change corresponds to the transition from one extended saddle point singularity to another one. In these regions the spectrum is almost one dimensional. At Fig.7 the energy in the normal state is presented as function of the corresponding momentum: $p_{x}$ for the a-vicinity, and $p_{y}$ for the b-vicinity (dashed curve). In order to obtain from here the superconducting spectrum one has to reflect the curve with respect to the chemical potential (straight horizontal line) and introduce gaps at the intersections. Then we get the two continuos curves at Fig.7. The momentum and energy lost by the neutron are absorbed by the electron system and can be described, as transitions from the lower to the upper curve. Singular points in the scattering probability as function of energy can appear, if both, the initial and the final point correspond to the maxima of the density of states, i.e. to the extrema of the curves. The lowest energy corresponds to the transition between the maximum of the lower curve (1) to the minimum of the upper (1'), and at $T=0$ this is the threshold, located at $2 \mathrm{D}$. Then comes the transition from the maximum of the lower curve (1) to the maximum of the upper (2'). Ths must be a maximum but it is not a threshold, since processes with smaller and larger energies are possible. Finally comes the transition from the minimum of the lower curve (2) to the maximum of the upper ( $\left.\mathrm{A}^{\prime}\right)$. This corresponds to the end of an absorption path, and hence 
the scattering probability must have a drop at this point. Rigorous calculations confirm this picture. The result is presented at Fig.8. This has to be compared with the experimental curve at Fig.9 [20]. It should be added that due to the so called "coherence factor" the maximum appears only, if the order parameter has different signs for the initial and final states (dwave symmetry). This makes the inelastic neutron scattering, as well as the negative isotope effect described above rather unique bulk measurements confirming the d-wave order parameter. The measurements associated with surfaces and interfaces can reveal properties different from the bulk, as, e.g., the suppression of the d-wave order parameter and appearance of a subdominant s-wave. Another important feature is that in a two-layered substance, which is YBCO, the maximum appears only in the channel which corresponds to the interaction of neutrons with the electron spin fluctuations antisymmetric with respect to the layers. Such fluctuations correspond to "acoustical" gapless spin-wave branch in the antiferromagnetic insulating phase. This is natural, since other fluctuations immediately decay into quasiparticles. On the other hand, such fluctuations are rather long ranged, and therefore they define, actually, the true "coherent" gap. It is no surprise, therefore, that with underdoping the position of the neutron maximum varies proportional to Tc and not to the gap in the quasiparticle spectrum measured in tunneling experiments.

\section{UPPER CRITICAL FIELD}

The last interesting consequence of the model, which I will describe, is the positive curvature of the upper critical magnetic field, as function of temperature [23] which was observed in many experiments, (e.g. [24, see Fig.10). The usual calculation gives a negative curvature. The reason for such a change is contained in the form of the spectrum. As it was mentioned before, the most important are the vicinities of the singular regions, where the motion of electrons becomes one-dimensional. There is, however, a hopping between the "a" and "b" vicinities, and hence a two-dimensional motion. Therefore, close to $T_{c}$ the field $H_{c 2}$ is defined by the usual orbital motion. With departing from Tc the situation changes. This can be seen from the Ginsburg-Landau type equations derived in the vicinity of $T_{c}$. They correspond to the minimum of the free energy

$$
\begin{array}{r}
F_{0}=\int d x d y\left\{\frac { 1 } { 4 m _ { x } } \left[\left|\left(-i \frac{\partial}{\partial x}-\frac{2 e}{c} A_{x}\right) \Psi_{a}\right|^{2}\right.\right. \\
\left.+\left|\left(-i \frac{\partial}{\partial y}-\frac{2 e}{c} A_{y}\right) \Psi_{b}\right|^{2}\right] \\
+\alpha\left[\tau\left(\left|\Psi_{a}\right|^{2}+\left|\Psi_{b}\right|^{2}\right)+\eta\left|\Psi_{a}+\Psi_{b}\right|^{2}\right. \\
\left.\left.+(1 / 2 n)\left(\left|\Psi_{a}\right|^{4}+\left|\Psi_{b}\right|^{4}\right)\right]+\frac{H^{2} d}{8 \pi}\right\}
\end{array}
$$

where

$$
\alpha=\frac{2\left(\pi T_{c}\right)^{2}}{7 \zeta(3) \mu_{1}}, \tau=\frac{T-T_{c}}{T_{c}}, \eta=\frac{\left|\lambda_{2}\right|}{\left(\lambda_{1}+\left|\lambda_{2}\right|\right)^{2}}
$$

The chemical potential $\mu_{1}$ is calculated with respect to the bottom of the band. The term with $\alpha \tau$ is negative below $T_{c}$ and it keeps $\Psi_{a, b}$ attached to a certain vicinity, whereas the term with $\alpha \eta$ corresponds to hopping between them. With increasing $|\tau|$ the motion becomes more one-dimensional, and eventually the orbital motion is unable to destroy superconductivity, i.e., $H_{c 2}$. For sufficiently small values of $\mathrm{h}$ this can happen even close to Tc (see Fig.11). In the immediate vicinity of Tc

$$
H_{c 2} \approx \frac{4 m c}{e} \alpha|\tau|\left(1+\frac{|\tau|}{\eta}\right)
$$

For sufficiently high fields another mechanism becomes important, namely the paramagnetic destruction of Cooper pairs. Unfortunately, the account of this effect leads to the restoration of a negative curvature at lower temperatures and larger fields. This does not fit the experimental data (see [25]).

There was an interesting suggestion [26] that in the cases of reduced dimensionality (films, wires in longitudinal field), where the orbital destruction becomes impossible, the paramagnetic effect leads to a sequence of phase transitions between different inhomogeneous LOFF (Larkin-Ovchinnikov-FuldeFerrell) phases with increasing critical fields. This is a possible origin of the positive curvature at very low temperatures.

\section{ACKNOWLEDGMENTS}

This work was supported by the Department of Energy under the contracts \# W-31-109-ENG-38.

[1] J. P. Franck et al., Physica B 169, 697 (1991); J. P. Franck et al., Phys.Rev. B 44, 5318 (1991).

[2] H. J. Bornemann a. D. E. Morris, Phys. Rev. B 44, 5322 (1991).

[3] N. Tralshavala et al., Phys. Rev. B 51, 3812 (1995).

[4] Y. Shina et al., Journ. Phys. Soc. Japan 64, 2577 (1995).

[5] Ya. G. Ponomarev, E. B. Tsokur, M. V. Sudakova, S. N. Tshesnokov, M. E. Shabalin, M. A. Lorenz, M. A. Hein, G. M'uller, H. Piel, B. A. Aminov, Solid. St. Comm. 111, 315 (1999).

[6] A. A. Abrikosov, J. C. Campuzano, A. K. Gofron, Physica C 214, 73 (1993); K. Gofron et al., Phys. Rev. Lett. 73, 3302 (1994)

[7] D. S. Dessau et al., Phys. Rev. Lett. 71, 2781 (1993)

[8] D. L. Novikov a. A. J. Freeman, Physica C 212, 233 (1993); ibid. 216, 273 (1993)

[9] A. A. Abrikosov, Physica C 244, 243 (1995)

[10] P. C. Pattniak et al., Phys. Rev. B 45, 5714 (1992)

[11] A. A. Abrikosov, Physica C 222, 191 (1994)

[12] A. A. Abrikosov, Phys. Rev. B 52, R15738 (1995)

[13] A. A. Abrikosov, L. P. Gor'kov, I. E. Dzyaloshinskii, Quantum Field Theory Methods in Statistical Physics, Pergamon, New York, 1965

[14] A. A. Abrikosov, Phys. Rev. B 53, R8910 (1996) 
[15] G. Giapintzakis, M. A. Kirk, D. M. Ginsberg (unpublished)

[16] M. Covington et al., Phys. Rev. Lett. 79, 277 (1997)

[17] A. A. Abrikosov (unpublished)

[18] T. Strach et al., Phys. Rev B 51, 16460, (1995)

[19] R. Henn et al., Phys. Rev. B 55, 3285 (1997)

[20] A. A. Abrikosov, Phys. Rev. B 57, 8656 (1998)

[21] P. Bourges in Proceedings of the NATO ASI summer school, 1997 (unpublished)

[22] J. M. Valles et al., Phys. Rev. B 44, 247 (1991)

[23] A. A. Abrikosov, Phys. Rev. B 56, 446 (1997)

[24] A. P. Mackenzie et al., Phys. Rev. Lett. 71, 1238 (1993)

[25] A. A. Abrikosov, Phys. Rev. B 56, 5112, (1997)

[26] A. I. Buzdin a. J. P. Brison, Europhys. Lett. 35, 707 (1996)

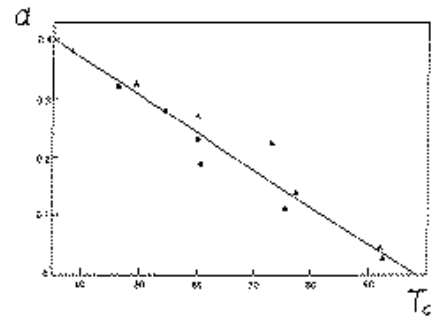

FIG. 1. Experimental results for the isotope shift $\left(O_{16} \rightarrow O_{18}\right)$ in partially substituted YBCO $(Y \rightarrow P r$, and $B a \rightarrow L a)$. The exponent $\alpha$ in $T_{c} \propto M^{\alpha}$ is presented as function of $T_{c}$ together with the linear regression.

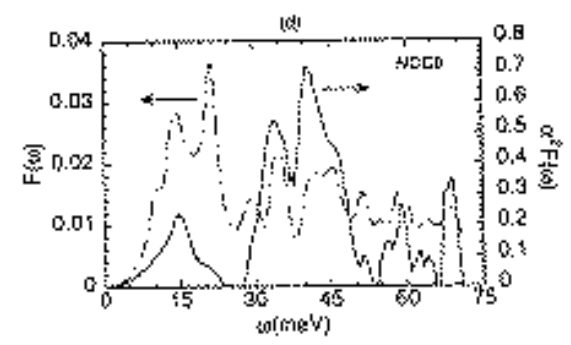

FIG. 2. Compilation of tunneling $\alpha^{2} F(\omega)$ for NCCO (solid line) and the phonon density of states $F(\omega)$ generated from the dispersion curves obtained from the inelastic neutron scattering from single-crystal NCCO

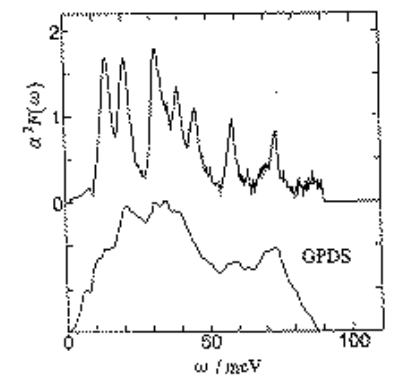

FIG. 3. The same, as in Fig. 2, for BSCCO. The lower curve is $F(\omega)$ from inelastic neutron scattering

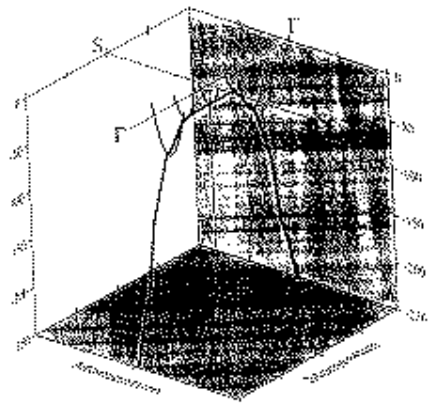

FIG. 4. ARPES result for the dependence of quasiparticle energy on components of quasimomentum in the vicinity of the extended saddle point singularity.

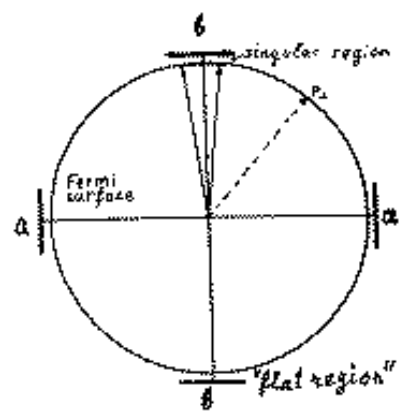

FIG. 5. Fermi surface with singular regions having a high density of states. A short range interaction connects any point with the singular region, whereas a long range interaction connects only points within this region.

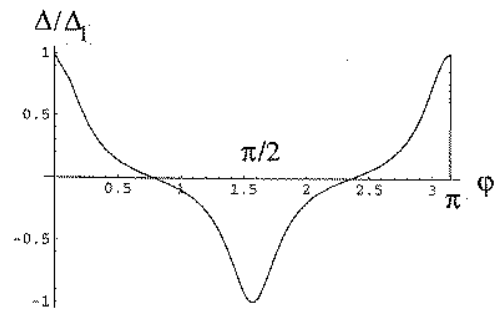

FIG. 6. Plot of $\Delta / \Delta_{1}$ according to eq. 5 .

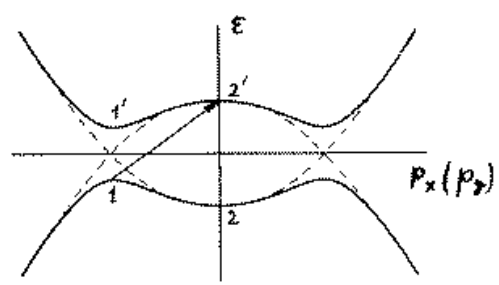

FIG. 7. Schematic presentation of the superconducting bands in the vicinity of singular points. The abscissa is $p-x$ for the a-vicinity, and $p_{y}$ for the b-vicinity. The notations $1,1^{\prime}, 2,2^{\prime}$ correspond to extremal points with the maximal density of states. 


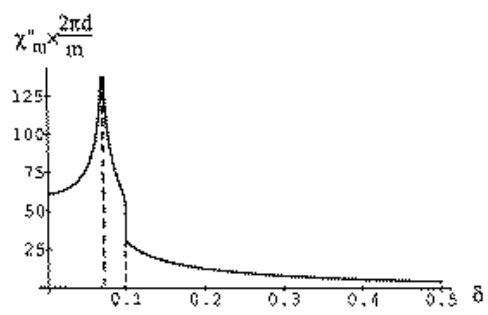

FIG. 8. Plot of the imaginary part of the electronic spin susceptibility, which is the energy dependent factor in the neutron inelastic scattering cross section $\chi_{m}^{\prime \prime} \times \frac{2 \pi d}{m}$ (m-effective mass, d-period along), as function of $\delta \equiv(\Omega / \Delta-2)^{1 / 2}$ where $\Omega$ is the energy loss, in the case $\gamma \equiv \mu_{1} / \Delta=0.1$ ( $\mu_{1}$ is the Fermi energy with respect to the bottom of the $1 \mathrm{D}$ band). Here the point $\Omega=2 \Delta$ is the threshold, and below this poind $\chi^{\prime \prime}=0$

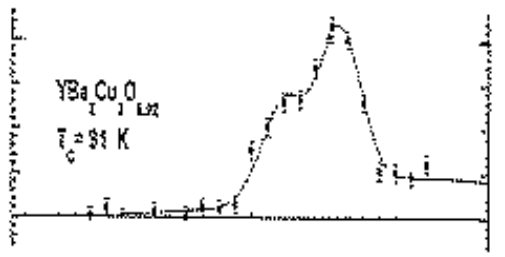

FIG. 9. Experimental data from [21] for the same quantity in arbitrary units as function of energy loss for optimally doped YBCO. The initial rise corresponds to the smeared out threshold.

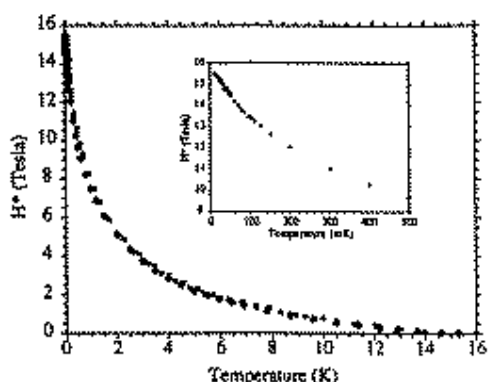

FIG. 10. Experimental results [24] for the temperature dependence of the upper critical field

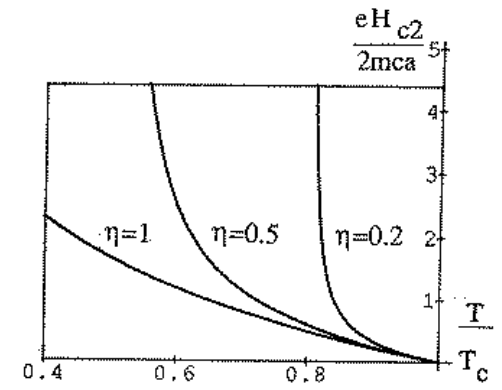

FIG. 11. Theoretical dependence of $e H_{c 2} /(2 m c \alpha)$ on $T / T_{c}$ for different values of $\eta$. 